\title{
NUMERICAL INVESTIGATION OF VAPOR BUBBLE GROWTH IN RECTANGULAR MICROCHANNEL
}

\author{
A. Stroh, ${ }^{1, *}$ B. Frohnapfel, ${ }^{1}$ R. Dittmeyer ${ }^{2}$ \\ ${ }^{1}$ Institute of Fluid Mechanics, Karlsruhe Institute of Technology, Karlsruhe, 76131, Germany \\ ${ }^{2}$ Institute for Micro Process Engineering, Karlsruhe Institute of Technology, Eggenstein-Leopoldshafen, \\ 76344, Germany
}

\begin{abstract}
A numerical simulation of a rectangular microchannel with partially superheated wall area is carried out in order to gain better understanding of the physical mechanism behind boiling in such confined geometries. Variation of the initial bubble position and boundary conditions for temperature and velocity fields is performed to extract the distinct influence on the vapor bubble growth. A significant difference in the motion and growth of the vapor bubble is observed depending on the operating conditions.
\end{abstract}

KEY WORDS: boiling and evaporation, heat exchanger, nano/micro scale measurement and simulation

\section{INTRODUCTION}

Efficient cooling in microscale devices often requires realization of large heat fluxes [1,2]. An interesting option in this respect are micro heat exchangers with phase change. Commonly, such heat exchanger includes a number of microchannels in which flow boiling occurs. While microchannels are filled with liquid through a flow distribution system, evaporation is expected to occur in each channel and the resulting gas is supposed to exit the channels through a common outlet. Experimental investigations of these devices reveal a highly dynamic process where the location of the phase change varies significantly in time and space [3-5]. This phenomena is accompanied by pulsating pressure, reversed flow or blockage of the microchannel-array. The current investigation is inspired by a series of experiments previously carried out at the Institute for Micro Process Engineering at KIT in Karlsruhe, Germany [6-8], which provide the necessary key data for the numerical setup. In order to gain a better physical understanding of the governing parameters of the boiling flow in the experimentally investigated microchannels we investigate the effects of the prescribed flow velocity, temperature distribution and initial position on the growth and motion of the vapor bubble using numerical simulation. Since a simulation of a complete microchannel-array is complex and extremely costly from the computational point of view, a single channel configuration is considered throughout the present manuscript.

\section{NUMERICAL PROCEDURE}

Numerical experiments are carried out based on the two-phase volume-of-fluid solver from the OpenFOAM software package extended by thermally driven two-phase flow phenomena [9]. The continuum method by Brackbill, Kothe \& Zemach [10] is used for surface tension modelling, while thermally driven phase change is

${ }^{*}$ Corresponding A. Stroh: stroh@kit.edu 
Table 1 Domain configuration.

\begin{tabular}{|ccc|}
\hline grid size & dimensions & resolution \\
$\left(N_{x} \times N_{y} \times N_{z}\right)$ & $\left(L_{x} \times L_{y} \times L_{z}\right)$ & $\Delta x, \Delta y, \Delta z$ \\
\hline $480 \times 40 \times 40$ & $1.2 m m \times 0.1 m m \times 0.1 m m$ & $2.5 \mu m$ \\
\hline
\end{tabular}

realized using the equilibrium interface model by Rattner \& Garimella [11]. The contact line dynamics of the phase fraction is modeled with a dynamic contact angle model based on the correlation by Kistler [12].

\subsection{Numerical Domain}

A single channel configuration is considered in a rectangular domain on an equidistantly spaced grid with resolution of $2.5 \mu \mathrm{m}$. The simulation is carried out using a symmetrical domain with a symmetry plane at $z=0.1 \mathrm{~mm}$, which results in the actual microchannel width of $0.2 \mathrm{~mm}$. The channel size corresponds to the smallest channels in the microchannel-array from the investigation performed by Maikowske, Brandner \& Lange [6]. Figure 1 and Table 1 summarize further details of the simulation setup. The flow is bounded by no-slip boundary condition applied on the side walls $\left(z=0,2 L_{z}\right)$, upper and bottom walls $\left(y=0, L_{y}\right)$, while a laminar flow profile is prescribed on the inlet plane of the channel $(x=0)$. The velocity distribution is given by the empirical function:

$$
u(x=0, y, z)=U_{\max }\left(1-\left|y-L_{y}^{2}\right|^{2.05}\right)\left(1-\left|z-L_{z}^{2}\right|^{3.0195}\right),
$$

which describes the laminar profile in a rectangular duct with ratio $L_{z} / L_{y}=2$ [13] with $U_{\max }$ being the centerine velocity. In the reference simulation the centerline velocity is chosen to be $U_{\max }=0.1 \mathrm{~m} / \mathrm{s}$, which corresponds to mean inlet velocity $\bar{U}=1 /\left(2 L_{y} L_{z}\right) \int_{y, z} u(x=0, y, z) \mathrm{d} y \mathrm{~d} z=0.05 \mathrm{~m} / \mathrm{s}$. Outlet boundary condition for velocity is set to zero-gradient. At the lower wall a non-uniform streamwise distribution of temperature $T_{w}(x)=T-T_{\text {sat }}$ is prescribed, where for $x>0.4 \mathrm{~mm}$ a constant $T_{w}=5 \mathrm{~K}$ is applied. Zero-gradient boundary conditions for $T$ are applied elsewhere. For the phase fraction field, $\alpha$, which varies from 0 (gas phase) to 1 (fluid phase), zero-gradient conditions are prescribed at the inlet and outlet of the domain, while a dynamic contact angle model by Kistler [12] is applied at the walls. Corresponding to the experiment [6], the lower wall and side-walls contact angles are set to $72^{\circ}$ and $40^{\circ}$ for advancing angle, $\theta_{a}$, and receding angle, $\theta_{r}$ (stainless steel). Since the upper wall represents a glass plate the advancing and receding angles are set to $30^{\circ}$ and $24^{\circ}$ on this boundary, respectively. Reference pressure is set to zero at the outlet plane, while zero-gradient condition is applied elsewhere. For investigation of the boundary condition effects on the development of the bubble growth, a variation of the inlet mean velocity, wall temperature distribution and contact angle properties is performed.

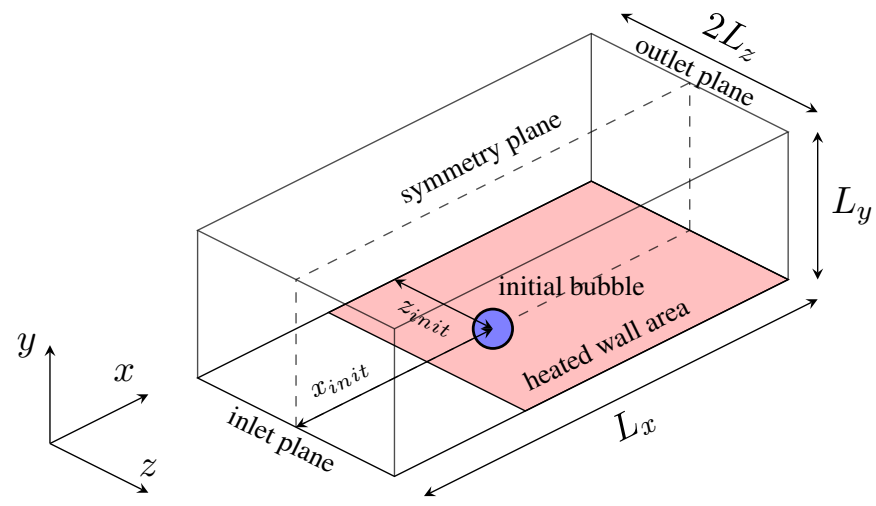

Fig. 1 Schematic of the numerical setup. 
Table 2 Properties of the phases at $T_{\text {sat }}=450 \mathrm{~K}$.

\begin{tabular}{|cccccc|}
\hline phase & $v\left[\mathrm{~m}^{2} / \mathrm{s}\right]$ & $\rho\left[\mathrm{kg} / \mathrm{m}^{3}\right]$ & $\lambda\left[\mathrm{kgm} / \mathrm{s}^{3} \mathrm{~K}\right]$ & $c_{p}\left[\mathrm{~m}^{2} / \mathrm{s}^{2} \mathrm{~K}\right]$ & $\sigma\left[\mathrm{kg} / \mathrm{s}^{2}\right]$ \\
\hline water & $1.71 \cdot 10^{-7}$ & 890.47 & 0.678 & 4400 & \multirow{2}{*}{0.0429} \\
vapor & $3.089 \cdot 10^{-6}$ & 4.808 & 0.0331 & 2560 & \\
\hline
\end{tabular}

\subsection{Initial Conditions}

The simulation is initialized with the constant laminar profile and fluid phase throughout the domain and runs single-phase until $t=0.02 \mathrm{~s}$ in order to obtain developed velocity and temperature fields. At the time instant $t=0.02 \mathrm{~s}$ an initial bubble is introduced in a form of a sphere $\left(r_{\text {init }}=0.02 \mathrm{~mm}\right)$ at the lower wall in the middle of the channel $\left(y_{\text {init }}=0, z_{\text {init }}=0.1 \mathrm{~mm}\right.$ ), where $\alpha$ and $T-T_{\text {sat }}$ within the sphere are set to zero. The streamwise position of the bubble, $x_{\text {init }}$, is varied in the numerical experiment. Table 2 provides a detailed list of phase properties (water and vapor at $T_{\text {sat }}=450 \mathrm{~K}$ ) used in the simulation.

\section{RESULTS}

In a first step, a variation of initial vapor bubble position is investigated. Figure 2 presents the temporal evolution of the mean phase fraction in streamwise, wall-normal and spanwise directions,

$$
\begin{gathered}
\alpha_{y z}(x, t)=1 /\left(2 L_{y} L_{z}\right) \int_{y, z} \alpha(x, y, z, t) \mathrm{d} y \mathrm{~d} z, \\
\alpha_{x z}(y, t)=1 /\left(2 L_{x} L_{z}\right) \int_{x, z} \alpha(x, y, z, t) \mathrm{d} x \mathrm{~d} z \\
\alpha_{x y}(z, t)=1 /\left(L_{x} L_{y}\right) \int_{x, y} \alpha(x, y, z, t) \mathrm{d} x \mathrm{~d} y,
\end{gathered}
$$

for $x_{i n i t}=0.60 \mathrm{~mm}$ (midpoint of the channel) and $x_{i n i t}=0.45 \mathrm{~mm}$ (at the edge of the heated area).

The case with initial bubble in the channel's midpoint demonstrates three distinct time periods where 3dimensional $(t<23 \mathrm{~ms})$, 2-dimensional $(23<t<26.5 \mathrm{~ms})$ and 1-dimensional $(t>26.5 \mathrm{~ms})$ bubble growth is observed. A transition between the periods occurs when the bubble exceeds the geometrical constraints of the microchannel, so an expansion in certain direction can not be maintained any more. Due to the aspect ratio of the channel, at first the upper wall is reached by the bubble within $3 \mathrm{~ms}$, while the side walls get in contact with gas phase $6.5 \mathrm{~ms}$ after initial bubble introduction. A notably interesting motion of the vapor bubble is observed: in the initial phase bubble moves upstream from the original position; after touching the upper wall the motion direction switches and the bubble travels downstream during the remaining simulation time. After the bubble attaches to the side-walls the streamwise position changes linearly, which corresponds to a constant bubble velocity $(\approx 0.06 \mathrm{~m} / \mathrm{s})$. Figure 3 shows the velocity field and streamlines at the symmetry plane $(z=0.1 \mathrm{~mm})$ for three time instants corresponding to three evolution phases. Velocity upstream of the bubble is barely affected by the growth for smaller bubble sizes, but the effect becomes visible at later time steps, when the bubble exceeds the dimensions of the channel. The plots reveal a very complex nature of the velocity distribution in the gas phase. Several vortical structures can be observed inside the bubble close to the contact line with upper and lower walls. While similar vortical structures are observed at $t=0.0202 \mathrm{~s}$ and $0.025 \mathrm{~s}$, the streamlines at $t=0.028$ show a different pattern, which implicates a reorganization of the flow topology inside the gas phase when bubble occupies the entire height and width of the channel.

The case with $x_{\text {init }}=0.45 \mathrm{~mm}$ shows a significantly different behavior. The bubble grows only for a very short time period $(\approx 1 \mathrm{~ms}$ after initial bubble placement) and then travels upstream without changing in size. Obviously, the initial upstream motion almost instantly removes the bubble from the thermal boundary layer of the heated area and evaporation process stops so the bubble dimensions remain constant. Thereby the maximum 

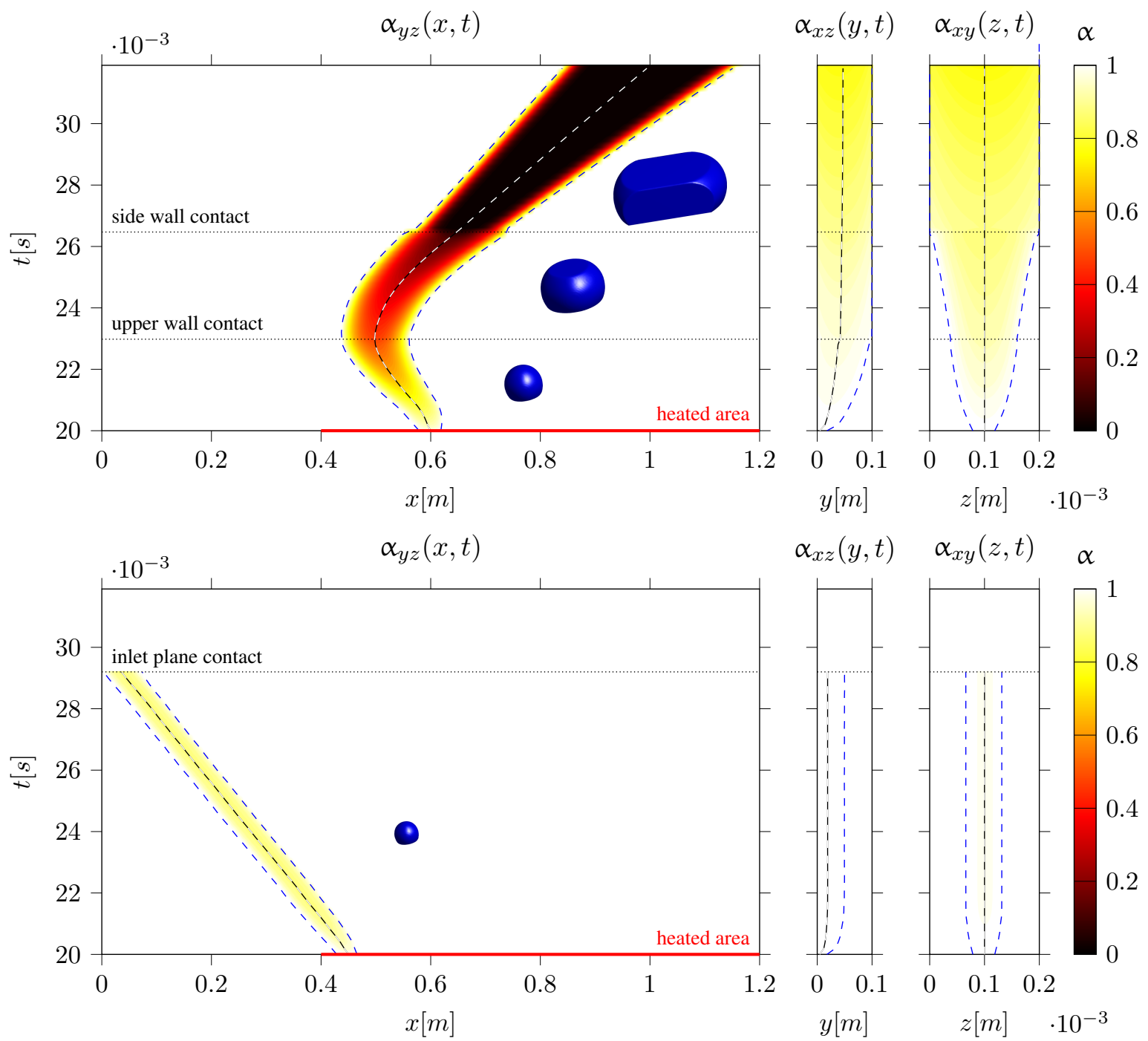

Fig. 2 Spatio-temporal evolution of phase fraction at $\bar{U}=0.05 \mathrm{~m} / \mathrm{s}$ in three channel directions with initial vapor bubble placement at $x_{i n i t}=0.60 \mathrm{~mm}$ (top picture) and $x_{i n i t}=0.45 \mathrm{~mm}$ (bottom picture). Dashed blue lines show the boundaries of the vapor bubble, while dashed black line marks the geometrical center of the bubble. Dotted lines mark the time of vapor bubble interaction with domain boundaries. Characteristic bubble shape is demonstrated for every time period of evolution.

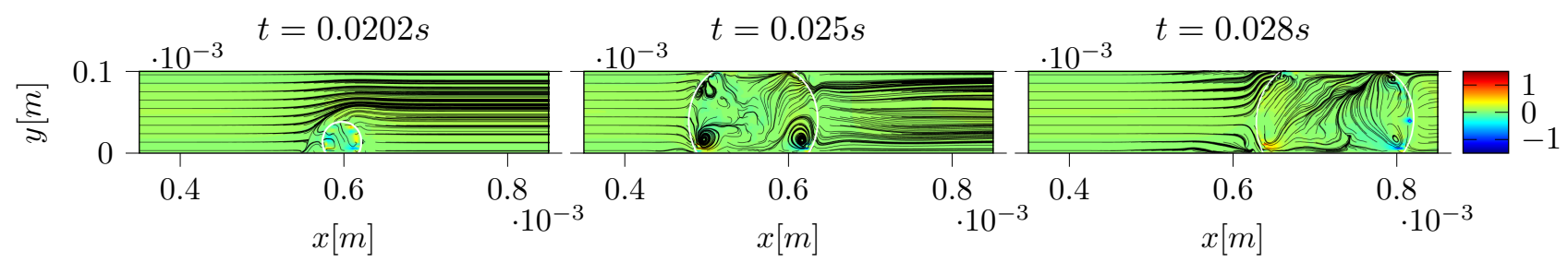

Fig. 3 Velocity distribution with streamlines in $x y$-plane at $z=0.1 \mathrm{~mm}$ for three time instants and initial bubble placement at $x_{\text {init }}=0.60 \mathrm{~mm}$. White line marks the phase interface. 
IHTC16-22961

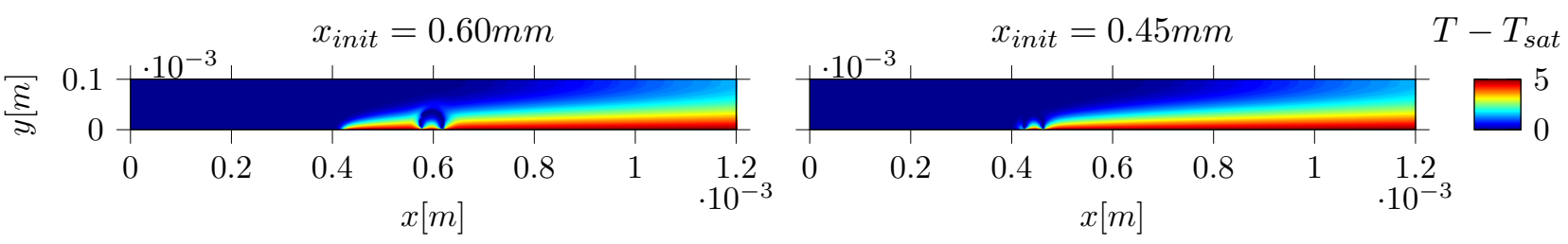

Fig. 4 Temperature distribution in $x y$-plane at $z=0.1 \mathrm{~mm}$ for two initial bubble placements at $t=0.0202 s$.

bubble diameter approaches the channel half height and a continuous upstream motion with $-0.04 \mathrm{~m} / \mathrm{s}$ occurs. Figure 4 shows the comparison of temperature distribution on the symmetry plane $z=0.1 \mathrm{~mm}$ for $x_{\text {init }}=$ $0605 \mathrm{~mm}$ and $0.45 \mathrm{~mm}$ right after initial bubble is introduced into the flow. From this distributions it is evident that the bubble placed closer to the edge of the heated area experiences lower temperature gradients and hence exhibits slower growth rate.

In a second step, a modification of the boundary conditions for both initial bubble placements is performed in order to understand the growth and motion evolution of the vapor bubbles observed in Fig. 2. The results of these modifications are shown in Fig. 5, where the streamwise mean phase fraction, $\alpha_{y z}$, is plotted as a function of time and compared to the reference simulations (second row). At first, an increase of the inlet mean velocity $\bar{U}$ to $0.25 \mathrm{~m} / \mathrm{s}\left(U_{\max }=0.5 \mathrm{~m} / \mathrm{s}\right)$ is considered (Fig. 5, first row). Qualitatively, the increase of inlet velocity doesn't change the bubble behavior, upstream motion can be still observed and the downstream motion occurs much faster. The bubble growth is very weak, which is linked to the presence of a thinner thermal boundary layer and stronger convective transport.

Reduction of the inlet velocity to zero allows us to see the vapor bubble growth without effects linked to the convection (Fig. 5, third row). It is evident that both initial bubble placements show a qualitatively similar evolution. In the case of $x_{\text {init }}=0.45 \mathrm{~mm}$ the bubble doesn't travel upstream of the initial position any more, which might be an indication that the upstream motion is caused by the presence of the inflow velocity. The slower bubble growth in this case is linked to the fact that the bubble placed closer to the heated area edge experiences lower temperature gradients and hence evaporation occurs slower.

Finally, to determine the influence of the temperature gradient at the lower wall the distribution of the wall temperature is switched to a uniform $T_{w}=5$ along the entire channel length. The inflow velocity is set to zero for this case. This allows us to identify the effect of streamwise temperature gradient on the vapor bubble growth. For the initial placement $x_{i n i t}=0.60 \mathrm{~mm}$ the results completely reproduces the result with the nonuniform temperature distribution, which confirms that the bubble behavior doesn't experience any effects from the streamwise inhomogeneity of the temperature field. The case with $x_{i n i t}=0.45 \mathrm{~mm}$ also shows exactly the same behavior, but shifted in space, which is expected since homogeneous boundary conditions are imposed in the streamwise direction.

\section{CONCLUSIONS}

A numerical simulation of vapor bubble growth in a rectangular microchannel with the dimensions of $1.2 \mathrm{~mm} \times$ $0.2 \mathrm{~mm} \times 0.1 \mathrm{~mm}$ is carried out with a volume-of-fluid solver. The lower wall of the microchannel is partially heated in the region $x>0.4 \mathrm{~mm}$, where a thermally driven vapor bubble growth is investigated. In the two reference simulations the position of the initial bubble is varied from $x_{\text {init }}=0.60 \mathrm{~mm}$ to $x_{\text {init }}=0.45 \mathrm{~mm}$. For $x_{\text {init }}=0.60 \mathrm{~mm}$ one can observe three time periods, where different bubble motion and growth is observed. First, bubble travels upstream, grows and touches the upper wall, after which a reverse of bubble motion occurs, so a downstream motions is observed from this time instant. After the bubble forms dry zones also at the channel side walls a downstream motion with constant velocity is observed. In the case when the bubble 


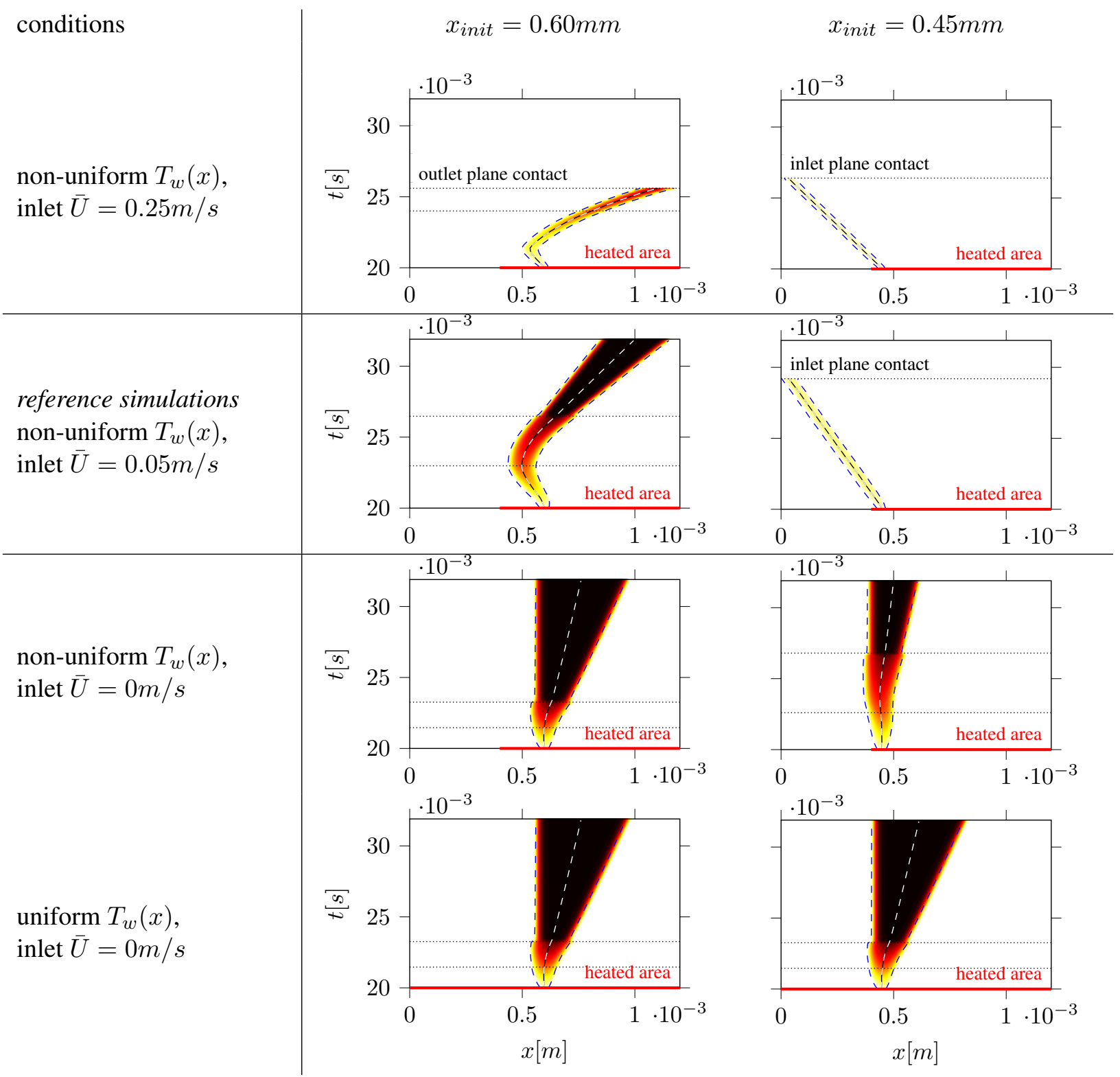

Fig. 5 Influence of boundary conditions on the spatio-temporal evolution of mean streamwise phase fraction, $\alpha_{y z}(x)$, with initial vapor bubble placement at $x_{\text {init }}=0.60 \mathrm{~mm}$ and $x_{i n i t}=0.45 \mathrm{~mm}$. Color code and line legend correspond to the ones of Fig. 2. 
is initially placed at $x_{i n i t}=0.45 \mathrm{~mm}$, only an upstream motions of the bubble can be observed. Due to this movement bubble is removed from the heated area and evaporations process stops. Variation of initial and boundary conditions reveals a very complex nature of the evaporation process inside of the microchannel. The investigation suggests that the upstream movement might be governed by the inflow velocity, while streamwise gradient of the temperature field influences the bubble growth rate. However, further analysis of the flow field, pressure and temperature is needed to shed light on the mechanism of vapor bubble growth in microstructured geometries. In future investigations we plan to extend the analysis to variation of contact angle (dynamic vs. constant) and consider cases without phase change for a better understanding of the flow physics.

\section{ACKNOWLEDGMENTS}

This work was performed on the computational resource ForHLR II funded by the Ministry of Science, Research and the Arts Baden-Wrttemberg and DFG ("Deutsche Forschungsgemeinschaft").

\section{REFERENCES}

[1] Prasher, R. S., Chang, J.-Y., Sauciuc, I., Narasimhan, S., Chau, D., Chrysler, G., Myers, A., Prstic, S., and Hu, C. (2005) Nano and Micro Technology-Based Next-Generation Package-Level Cooling Solutions.. Intel Technology Journal, 9(4).

[2] Hetsroni, G., Mosyak, A., Pogrebnyak, E., and Segal, Z. (2006) Periodic boiling in parallel micro-channels at low vapor quality. International Journal of Multiphase Flow, 32(10), 1141-1159.

[3] Hetsroni, G., Mosyak, A., Segal, Z., and Pogrebnyak, E. (2003) Two-phase flow patterns in parallel micro-channels. International Journal of Multiphase Flow, 29(3), 341-360.

[4] Kandlikar, S. G., Kuan, W. K., Willistein, D. A., and Borrelli, J. (2006) Stabilization of flow boiling in microchannels using pressure drop elements and fabricated nucleation sites. Journal of Heat Transfer, 128(4), 389-396.

[5] Kakac, S. and Bon, B. (2008) A review of two-phase flow dynamic instabilities in tube boiling systems. International Journal of Heat and Mass Transfer, 51(3), 399-433.

[6] Maikowske, S., Brandner, J., and Lange, R. (2010) A novel device for the optical investigation of phase transition in micro channel array evaporators. Applied Thermal Engineering, 30(13), 1872-1876.

[7] Anurjew, E., Hansjosten, E., Maikowske, S., Schygulla, U., and Brandner, J. J. (2011) Microstructure devices for water evaporation. Applied Thermal Engineering, 31(5), 602-609.

[8] Brandner, J. J., Kamaruzaman, N., and Maikowske, S. (2011) A microstructure device for single phase surface cooling. In Proc. ASME/JSME 2011 8th Thermal Engineering Joint Conference AJTEC2011 pp. AJTEC2011AJTEC44038.

[9] Nabil, M. and Rattner, A. S. (2016) interThermalPhaseChangeFoam - A framework for two-phase flow simulations with thermally driven phase change. SoftwareX, 5, 216-226.

[10] Brackbill, J., Kothe, D. B., and Zemach, C. (1992) A continuum method for modeling surface tension. Journal of computational physics, 100(2), 335-354.

[11] Rattner, A. S. and Garimella, S. (2014) Simple mechanistically consistent formulation for volume-of-fluid based computations of condensing flows. Journal of Heat Transfer, 136(7), 071501.

[12] Berg, J. (1993) Wettability. New York, p. 75.

[13] Nataraja, N. M. and S., L. (1973) Laminar-flow in rectangular ducts-prediction of velocity profiles and friction factor. Indian Journal of Technology, 10(12), 435-438. 